\title{
Coordinated Planning of Electricity/gas/storage Distribution Network Based on LSTM and Demand Response
}

\author{
Yongming $\mathrm{Xu}^{1}, \mathrm{Xi} \mathrm{Ding}^{2 *}$, Jianxun $\mathrm{Xu}^{1}$, Yunqian $\mathrm{Li}^{1}$, Xueyu $\mathrm{Ma}^{1}$ and Chuangxin $\mathrm{Guo}^{2}$ \\ ${ }^{1}$ State Grid Zhejiang Jiashan Country Power CO.LTD, Jiashan, Zhejiang, 314100, China \\ ${ }^{2}$ College of Electrical Engineering, Zhejiang University, Hangzhou, Zhejiang, 310027, China
}

\begin{abstract}
This paper presents a collaborative planning method of an electricity-gas-storage regional integrated energy system based on LSTM neural network and demand response. First, the LSTM Neural network is used for load forecasting, and the energy hub structure of the electric gas storage system is established. Then, the mathematical models of power storage, gas storage, electric network topology, gas network topology, and $\mathrm{P} 2 \mathrm{G}$ are established to minimize the expansion cost of the electricity-gas-storage system, and the collaborative planning of energy storage, power lines, and natural gas pipelines is proposed based on the existing electric gas coupling integrated energy system. The original model which is difficult to solve is transformed into a mixed-integer linear programming model by introducing auxiliary variables, and the CPLEX solver is called to solve it. Finally, the economic advantages of collaborative planning of electricity-gas-storage system are verified by an example, and the connection of power storage and gas storage can reduce system pressure and optimize equipment selection.
\end{abstract}

\section{Introduction}

At present, the power demand of the user side is rising, while the supply side capacity expansion problem cannot be solved effectively in a short time. Therefore, the demand side resources with relatively low cost are paid more and more attention[1].Many scholars have carried out multi-directional research on demand-side load forecasting and proposed a variety of forecasting methods, such as linear extrapolation method, regression analysis method, time series method, wavelet analysis method, artificial neural network method, support vector machine method, and combination forecasting method [2-5].

With the gradual deepening of the coupling of electricity and gas resources, the comprehensive joint planning of electricity-gas-storage increases the interoperability between networks to a certain extent and reduces the total cost. Therefore, it is necessary to study the planning method of a regional integrated energy system [6-9].

[10] considers the investment cost, operation cost, and reliability cost, optimizes the regionally integrated energy system with electricity to gas device from the perspective of the optimal economy; in [11], considers the collaborative expansion of wind farm and $\mathrm{P} 2 \mathrm{G}$ equipment, and studies its impact on the system expansion scheme and wind power consumption.

However, there are few studies on the optimal allocation model of various devices in the electric gas multi-energy storage system, such as energy storage battery, gas pipeline, gas storage equipment, P2G equipment, and there is no unified planning combined with power load forecasting technology.

Based on the LSTM model and demand response, this paper proposes a collaborative planning method for the electricity-gas-storage regional integrated energy system. Firstly, load forecasting is carried out based on LSTM neural network. Then, the energy hub structure of the electricity-gas-storage regional integrated energy system is constructed, and the collaborative planning method after adding energy storage equipment based on the existing electric gas coupling system is analyzed. Then, the mathematical model of the gas network topology is transformed into the mixed-integer linear programming problem which is easy to solve by introducing the original nonlinear programming model into the auxiliary variables. Finally, the result is solved by calling CPLEX.

\section{Load forecasting technology based on LSTM}

Long short-term memory (LSTM) is a kind of time cycle neural network. It is an improved recurrent neural network (RNN). LSTM is a variant of the recurrent neural network, and it is also a chain structure, but there are some differences in repetitive modules. There is only one neural network layer in the repetitive module of the standard recurrent neural network, while LSTM has four network layers, which interact in a special form.

The core of LSTM is to introduce a new cell state, which transmits the linear cycle information and outputs the information to the hidden state at the same time. As shown in Figure 1, the cell state is the horizontal line

\footnotetext{
"Corresponding author's e-mail: 22060117@zju.edu.cn
} 
running through the whole repeating structure. In the figure, $\sigma$ and tanh represent sigmoid and tanh activation functions respectively, $x_{t}$ is input quantity, $h_{t}, h_{t-1}$ is hidden state of the current and previous time step, $i_{t}, f_{t}, o_{t}$ represent input gate, forgetting gate, and output gate at the current time respectively.

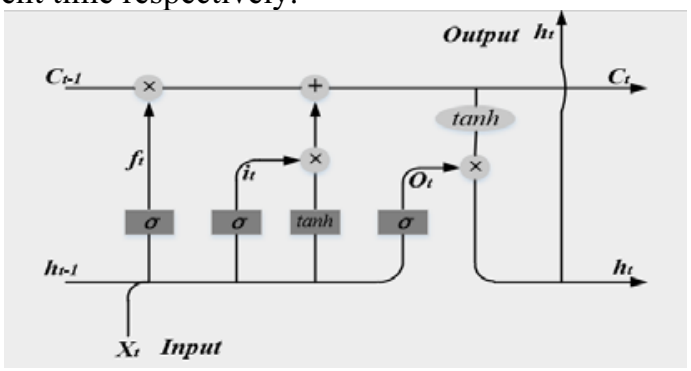

Figure 1. Long short-term memory neural network model

LSTM modeling process is as follows:

(1) Firstly, the input load is decomposed into different frequency components.

(2) The load component data obtained after decomposition is preprocessed, and the data is normalized.

(3) The parameters of the network are set to train the LSTM model.

The predicted load curve is shown in Figure 2.

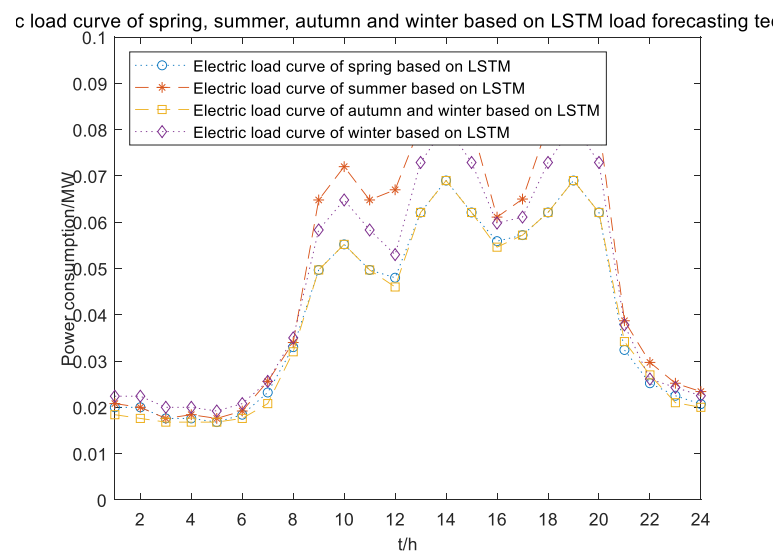

load curve of spring, summer, autumn and winter based on LSTM load forecasting tect

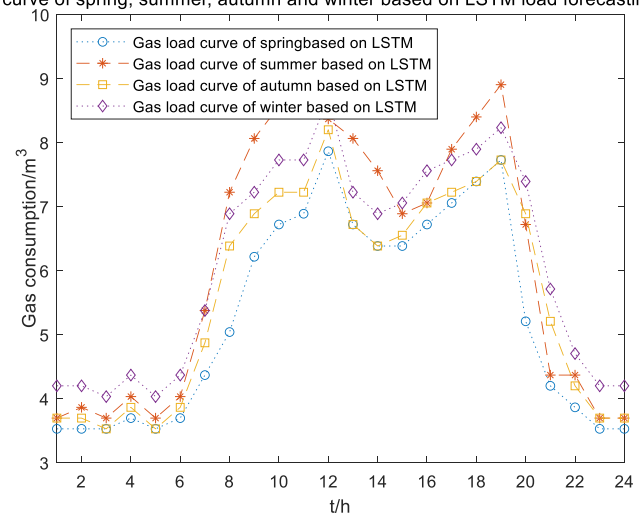

Figure 2. Electric and gas load curve of spring, summer, autumn and winter based on LSTM load forecasting technology

\section{Overall description of planning problems}

To better describe the characteristics of the electric-gasstorage integrated energy system, the energy hub structure is established, as shown in Figure 3.

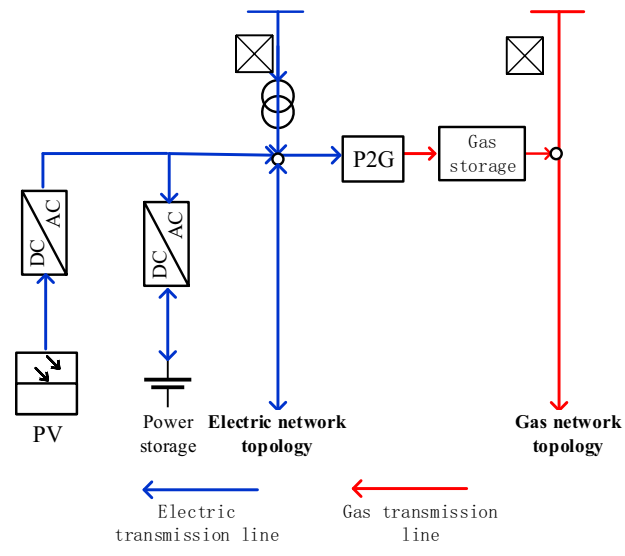

Figure 3. EH structure with electricity-gas-storage

The load characteristics of a regional integrated energy network will affect the optimal configuration results of the energy storage system. At the same time, access to energy storage will affect the line construction and equipment type selection in electric network topology and gas network topology to a certain extent. Therefore, it is necessary to carry out collaborative planning for the electricity-gas-storage regional integrated energy system.

\section{4 demand response}

\subsection{The Type of Demand Response}

The demand-side response is usually used in the time of use (TOU) system. In this paper, two types of demand response, load reduction and load transfer are considered. In the process of load transfer, the total load should remain unchanged, that is:

$$
\sum_{h} P_{m o v, y s h, i}=0 \quad \forall i \in \Omega_{m o v}, \forall y, \forall s, \forall h
$$

Where $i$ is the node number of the distribution network; y, s, h are the year, quarter, and hour respectively; $\Omega_{\text {mov }}$ is the set of transferable load nodes participating in the demand response; $P_{\text {mov }, y s h, i}$ is the transferred load of $i$ in the $h$ hour of the s quarter of the y year.

\subsection{User compensation cost}

If the demand response is brought into the planning model, the user compensation cost $C_{5}$ caused by the decrease of user comfort and flexibility should be considered.

$$
\begin{gathered}
C_{5}=\sum_{y} \sum_{s} \sum_{h} 1 /(1+d)^{y-1}\left(\sum_{i \in \Omega_{c u t}} C_{c u t} P_{c u t, y s h, i}+\right. \\
\left.\sum_{i \in \Omega_{\text {mov }}} C_{\text {mov }}\left|P_{\text {mov }, y s h, i}\right|\right)
\end{gathered}
$$

Where $C_{c u t}$ is the unit compensation cost of load reduction, $C_{m o v}$ is the unit compensation cost of load transfer, $P_{c u t, y s h, i}$ is the total load reduced by the node in the yth year, sth quarter, and hth hour, $P_{\text {mov,ysh }, i}$ is the total load transferred, $\Omega_{\text {cut }}$ is the collection of reducible 
load nodes participating in demand response, and the $\Omega_{\text {mov }}$ is the collection of transferable load nodes.

\subsection{Demand-response constraints considering user satisfaction}

In the implementation process of demand response, the less the load is reduced or transferred, and the shorter the average transfer time is (that is, the faster the transfer speed is), the higher the user satisfaction is.

\subsubsection{Transferable load constraint}

$$
\left|P_{m o v, y s h, i}\right| \leq \sigma_{1} D_{P, y s h, i} \quad \forall i \in \Omega_{m o v}, \forall y, \forall s, \forall h
$$

Where $D_{P, y s h, i}$ represents the original load when the node does not implement demand-side response and $\sigma_{1}$ represents the maximum proportion of transfer load to the original load.

\subsubsection{Reducible load constraint}

$$
0 \leq P_{c u t, y s h, i} \leq \sigma_{2} D_{P, y s h, i} \forall i \in \Omega_{c u t}, \forall y, \forall s, \forall h
$$

Where $\sigma_{2}$ represents the maximum proportion of the reduced load to the original load.

\section{Collaborative planning model and algorithm}

\subsection{Objective Function}

The investment subject of the collaborative planning model of electricity, gas, and storage proposed in this paper is the integrated energy service provider, and the objective function is to minimize the total cost of the coupled network system of electricity, gas, and storage under the condition of meeting the energy demand of users:

$$
\min C=C_{1}+C_{2}+C_{3}+C_{4}+C_{5}
$$

Where $C_{1}$ is the investment cost, $C_{2}$ is the maintenance cost, $C_{3}$ is the transaction cost of purchasing electricity/gas from the superior network, and $C_{4}$ is the investment cost of energy storage.

In this paper, the whole planning process is divided into two stages. In the first stage of planning, the formula is

$$
\begin{gathered}
C_{1}=\sum_{y} \sum_{f \in \Omega_{S}} \sum_{\theta \in \Omega_{\mathrm{TYPE}}} \frac{C_{\mathrm{inv}, f, \theta} x_{y, f, \theta}}{(1+d)^{y-1}} \\
C_{2}=\sum_{y} \sum_{f \in \Omega_{S}} \sum_{\theta \in \Omega_{\mathrm{TYPE}}} \frac{C_{\mathrm{mnt}, f, \theta} X_{y, f, \theta}}{(1+d)^{y-1}}
\end{gathered}
$$

$C_{3}$

$$
\begin{aligned}
& =\sum_{y} \sum_{s} \sum_{\mathrm{h}} \frac{1}{(1+d)^{y-1}}\left(\sum_{a \in \Omega_{\mathrm{SUB}}} \sum_{b, i, i) \in \Omega_{\mathrm{EL}}} C_{\mathrm{BUY}, \mathrm{e}} P_{e, y s \mathrm{~s}, a i}\right. \\
& \left.+\sum_{b \in \Omega_{\mathrm{GATE}}} \sum_{(b, m) \in \Omega_{\mathrm{GL}}} C_{\mathrm{BUY}, \mathrm{g}} F_{g, y s h, b m}\right)
\end{aligned}
$$

Where logical variables $X_{y, f, \theta}$ and $x_{y, f, \theta}$ are respectively $0-1$ variables indicating whether the equipment selection exists in y year and whether it is put into construction at the beginning of the year, $C_{i n v, f, \theta}$ and $C_{\mathrm{mnt}, f, \theta}$ are the investment cost and maintenance cost the selection $\theta$ of the equipment $f$ to be built, respectively. $C_{\mathrm{BUY}, \mathrm{e}}$ and $C_{\mathrm{BUY}}$ are the unit purchase cost of power and natural gas, respectively. $a$ and $i$ are the topology nodes of the regional power network, $b$ and $m$ are the topology nodes of the regional gas network. $P_{e, y s h, a i}$ and $F_{g, y s h, b m}$ are respectively the tidal current of power line $a i$ and natural gas pipelines $b m$ in year $\mathrm{y}$, quarter s, hour h, $\Omega_{\mathrm{SUB}}, \Omega_{\mathrm{GATE}}, \Omega_{\mathrm{EL}}, \Omega_{\mathrm{GL}}$ are respectively the collection of substations nodes, natural gas station nodes, power lines, and natural gas pipelines.

In the second stage of planning, the investment planning of multi-energy storage in an integrated energy network mainly includes two parts: energy storage battery and gas storage device.

$$
\begin{gathered}
C_{4}=k_{1} P_{e}^{\text {max }}+k_{2} C_{e}^{\max }+k_{3} P_{P 2 G}^{\max }+k_{4} C_{G S}^{\max }+ \\
k_{5}\left(P_{\text {gas.in }}^{\max }+P_{\text {gas.out }}^{\max }\right)
\end{gathered}
$$

Where, $P_{e}^{\max }$ and $C_{e}^{\max }$ respectively represent the maximum power and capacity of the storage battery, $P_{P 2 G}^{\max }$ are the maximum power of the P2G equipment, $C_{G S}^{\max }$ are the maximum capacity of the gas storage device, and $P_{\text {gas.in }}^{\max }, P_{\text {gas.out }}^{\max }$ are the maximum power of the input and output gas storage device respectively; Approximately think $P_{\text {gas.in }}^{\max }=P_{P 2 G}^{\max }$, $k_{1}, k_{2}$ represent unit power investment coefficient and unit capacity investment coefficient of storage battery respectively; $k_{3}$ represent unit power investment coefficient of P2G equipment; $k_{4}$ represent unit capacity investment coefficient of the gas storage device; $k_{5}$ represent unit power investment coefficient of gas transmission pipeline.

\subsection{Constraints}

\subsubsection{Energy storage constraints}

(1) Energy storage battery constraints

$$
\begin{gathered}
S O C_{s, t}^{e}=\left(1-\delta_{e}\right) S O C_{s, t-1}^{e}+\Delta t\left(\eta^{e, C} P_{s, t}^{e, C}-\frac{P_{s, t}^{e, F}}{\eta^{e, F}}\right) / C_{e}^{\max } \\
S O C_{\min }^{e} \leq S O C_{s, t}^{e} \leq S O C_{\max }^{e} \\
0 \leq P_{s, t}^{e, C}, P_{s, t}^{e, F} \leq P_{e}^{\max } \\
P_{s, t}^{e, C} \cdot P_{s, t}^{e, F}=0
\end{gathered}
$$

Where: $P_{e}^{\max }, C_{e}^{\max }$ denote the power configuration and capacity configuration of the energy storage battery respectively; $S O C_{s, t}^{e}$ denote the state of charge of the energy storage battery in t period; $\eta_{i}^{e, C}, \eta_{i}^{e, F}$ denote the charging and discharging efficiency of the energy storage battery $\mathrm{i}$ respectively; $\delta_{e}$ denote the self-discharging efficiency of the energy storage battery; $S O C_{i, \max }^{e}$, $S O C_{i, \mathrm{~min}}^{e}$, denote the upper and lower limits of the state of charge of the energy storage battery; $P_{s, t}^{e, C}, P_{s, t}^{e, F}$ denote the 
charging and discharging power in t period; $\Delta t$ denotes the time interval. $\Delta t$ takes one hour.

(2) Constraints of the gas storage system

$$
\begin{aligned}
& \operatorname{SOC}_{\mathrm{s}, \mathrm{t}}^{\mathrm{g}}=\left(1-\delta_{\mathrm{g}}\right) \mathrm{SOC}_{\mathrm{s}, \mathrm{t}-1}^{\mathrm{g}}+\frac{\Delta \mathrm{t}\left(\eta \eta^{\left.\mathrm{g}, \mathrm{C}_{\mathrm{s}, \mathrm{t}}^{\mathrm{g}, \mathrm{C}}-\frac{\mathrm{P}_{\mathrm{s}, \mathrm{t}}^{\mathrm{g}, \mathrm{F}}}{\eta \mathrm{g}, \mathrm{F}}\right)}\right.}{\mathrm{C}_{\mathrm{GS}}^{\max }} \\
& S O C_{\text {min }}^{g} \leq S O C_{s, t}^{g} \leq S O C_{\text {max }}^{g} \\
& 0 \leq P_{s, t}^{g, C} \leq P_{\text {gas.in }}^{\max } \\
& 0 \leq P_{s, t}^{\mathrm{g}, \mathrm{F}} \leq P_{\text {gas.out }}^{\max } \\
& P_{\text {gas.in }}^{\max }=P_{P 2 G} \\
& P_{P 2 G}=\eta_{P 2 G} \cdot P_{P 2 G}^{i n} \\
& 0 \leq P_{P 2 G} \leq P_{P 2 G}^{\max }
\end{aligned}
$$

Where $P_{P 2 G}^{\max }$ represents the maximum power of the P2G equipment; $C_{G S}^{\max }$ represents the maximum capacity of the gas storage device; $P_{\text {gas.in }}^{\max }, P_{\text {gas.out }}^{\max }$ represents the maximum power of the input and output gas storage devices respectively; Approximately think $P_{\text {gas.in }}^{\max }=$ $P_{P 2 G}^{\max } ; S O C_{s, t}^{g}$ represents the state of charge of the gas storage system; $\eta^{g, C}, \eta^{g, F}$ represents the charging and discharging efficiency of the energy storage battery during operation; $\delta_{g}$ represents the self-consumption efficiency of the gas storage system; $S O C_{\text {min }}^{g}, S O C_{\text {max }}^{g}$ represent the upper and lower limits of the state of charge of the gas storage system; $S O C_{s, t-1}^{g}$ represents the state of charge of the gas storage system at the end of period $t-1$; $P_{s, t}^{g, C}, P_{s, t}^{g, F}$ respectively represent the gas storage and gas consumption power in period $\mathrm{t} ; P_{P 2 G}^{i n}$ is the electrical power input by the $\mathrm{P} 2 \mathrm{G}$ equipment.

\subsubsection{Topological constraints of the power network}

(1) For any node, the total input and output of power flow keep balance.

$$
\begin{gathered}
\sum_{(i, j) \in \Omega_{\mathrm{EL}}} P_{e, y s \mathrm{~h}, i j}=\sum_{(k, i) \in \Omega_{\mathrm{EL}}} P_{e, y s \mathrm{~s}, k i}+P_{P 2 G, y s h, i} \\
\forall i \in \Omega_{\mathrm{EBUS}}, \forall y, \forall s, \forall \mathrm{h} \quad(21)
\end{gathered}
$$

Where $i, j, k$ is the node of electric network topology, $P_{e, y s h, i j}$ and $P_{e, y s h, k i}$ represent the power flow of power line and hour in the yth year, sth quarter, and hth hour respectively, $P_{P 2 G, y s h, i}$ is the output power of $\mathrm{P} 2 \mathrm{G}$ equipment at the node $i$ in the yth year, sth quarter, and hth hour, and $\Omega_{\mathrm{EBUS}}$ is the collection of electric network nodes.

(2) For each power line, there are power flow constraints

$$
0 \leq P_{e, y s h, i j} \leq z_{e, y s h, i j} P_{\mathrm{el}, \max , i j} \forall y, \forall s, \forall \mathrm{h}
$$

Where $P_{\mathrm{el}, \max , i j}$ is the upper limit value of power flow of power line $i j ; 0-1$ decision variable $z_{e, y s h, i j}$ is the direction auxiliary variable of power line $i j$; specifically, when there is power flow from node $i$ to node $j$ on the line, the value is 1 ; when there is no direct power flow from node $i$ to node $j$ on the line, the value is 0 . It should be noted here that $z_{e, y s h, i j}$ and $z_{e, y s h, j i}$ jointly represent the power flow direction of the line from two directions.
(1) For any natural gas node, the balance between the total power flow input and the total power flow output can be expressed as follows:

$$
\begin{array}{r}
\sum_{(m, n) \in \Omega_{\mathrm{GL}}} F_{g, y s h, m n}=\sum_{(l, m) \in \Omega_{\mathrm{GL}}} F_{g, y s h, l m}- \\
F_{P 2 G, y s h, m}-D_{G, y s h, m} \quad \forall m \in \Omega_{\mathrm{GBUS}}, \forall y, \forall s, \forall \mathrm{h}
\end{array}
$$

Where $m, n, l$ is the natural gas network node; $F_{g, y s h, m n}$ and $F_{g, y s h, l m}$ is the tidal current of the natural gas pipeline $m n$ and $l m$ in the yth year, sth quarter, and hth hour respectively; $F_{\text {cchp, } y s h, m}$ is the gas consumption flow of the $\mathrm{P} 2 \mathrm{G}$ equipment at the node $m$ in the yth year, sth quarter, and hth hour; $D_{G, y s h, m}$ is the natural gas load demand of the node $m$ in the yth year, sth quarter, and hth hour; $\Omega_{\mathrm{GBUS}}$ is the gas distribution network node aggregation.

(2) The relationship between node pressure and pipeline current is as follows $\left[\operatorname{sign}\left(F_{g, y s h, m n}-F_{g, y s h, n m}\right)\right]\left(F_{g, y s h, m n}-F_{g, y s h, n m}\right)^{2}=$
$C_{m n}{ }^{2}\left(p_{y s h, m}^{2}-p_{y s h, n}^{2}\right) \forall(m, n) \in \Omega_{\mathrm{GL}}, \forall y, \forall s, \forall \mathrm{h}(24)$

Where $\operatorname{sign}\left(F_{g, y s h, m n}-F_{g, y s h, n m}\right)$ represents the direction of tidal current in the natural gas pipeline $m n$; $p_{y s h, m}$ and $p_{y s h, n}$ represents the pressure of the two nodes $m$ and $n$ of the corresponding line respectively; $C_{m n}$ represents the constant of natural gas pipeline $m n$.

\section{Case analysis}

In this section, three planning scenarios are set, and the proposed method is applied to an actual Park in Zhejiang Province. Compared with the planning results, the superiority of the proposed method is verified, and the impact of energy storage access on the joint planning of the power gas distribution network is analyzed.

\subsection{Example data}

The topology of 12 nodes electric network, 10 nodes natural gas network, and user load in a certain area of Zhejiang Province are shown in Figure 4. The electricity gas network is coupled with each other through the $\mathrm{P} 2 \mathrm{G}$ equipment to be built.

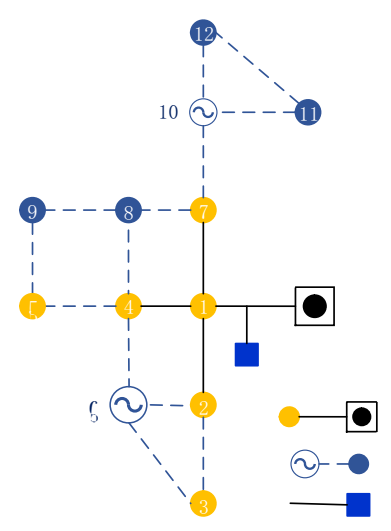

Subs tat ion nodes and existing line of the superior distribution network Loads to be built, distribution lines, $\mathrm{P} 2 \mathrm{G}$ equipment Power storage equipment to be built

\subsubsection{Topological constraints of gas network}




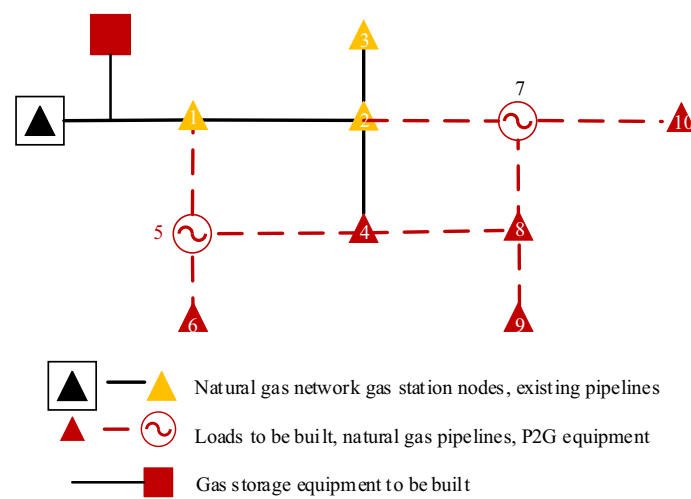

Figure 4. The topology of the 12-bus power distribution grid and 10-node gas distribution grid

The example uses the peak valley TOU price of Zhejiang Province, as shown in Table 1. According to the unified price of natural gas network 3yuan $/ \mathrm{m}^{3}$, the heating value coefficient is $9.7 \mathrm{kw} / \mathrm{m}^{3}$

Table 1. Time-of-use price

\begin{tabular}{|c|c|c|}
\hline $\begin{array}{c}\text { Peak and valley } \\
\text { category }\end{array}$ & Period & $\begin{array}{c}\text { electricity price / } \\
\left(\text { yuan } \cdot(\boldsymbol{k W} \cdot \boldsymbol{h})^{-\mathbf{1}} \text { ) }\right.\end{array}$ \\
\hline Peak & $08: 00-22: 00$ & 0.5583 \\
\hline $\mathrm{Gu}$ & $22: 00-8: 00$ & 0.3583 \\
\hline
\end{tabular}

Besides, The energy storage equipment in Zhejiang energy storage market is selected, and the main component parameters of lithium battery and gas storage tank are given.

Table 2. Main component parameters of energy storage

\begin{tabular}{|c|c|}
\hline System parameter & values \\
\hline Unit power cost of lithium battery (yuan / kW) & 400 \\
\hline Unit capacity cost of lithium battery (yuan / kWh) & 200 \\
\hline Unit power cost of the gas tank (yuan / kW) & 125 \\
\hline $\begin{array}{c}\text { Unit capacity cost of the gas storage tank (yuan / } \\
\text { kWh) }\end{array}$ & 200 \\
\hline Charging efficiency of energy storage & 0.90 \\
\hline $\begin{array}{c}\text { Energy storage discharge efficiency } \\
\text { Maximum allowable state of charge of energy } \\
\text { storage }\end{array}$ & 0.88 \\
\hline Minimum allowable state of charge of energy \\
storage
\end{tabular}

To verify the applicability of the proposed planning method to the collaborative planning of power gas storage integrated energy system and the impact of energy storage access on the planning results, a design example scenario is as follows:

Scenario 1: independent planning of electric network and natural gas network;

Scenario 2: collaborative planning of electricity and gas system coupled by $\mathrm{P} 2 \mathrm{G}$;
Scenario 3: collaborative planning of power gas storage regional integrated energy system coupled by $\mathrm{P} 2 \mathrm{G}$ after power storage and gas storage are connected.

\subsection{Results and analysis of an example}

Call CPLEX solver to get the optimal planning scheme under three scenarios, as shown in Table 3 . The planning results of power lines and natural gas pipelines are given in the form of "initial node terminal node (investment type)", and the planning results of $\mathrm{P} 2 \mathrm{G}$ are given in the form of "local power network node (investment type)", with 1-3 representing three types of equipment with capacity from small to large.

Table 3. Optimal planning schemes in three scenes

\begin{tabular}{|c|c|c|c|}
\hline scene & Power line & $\begin{array}{c}\text { Natural gas } \\
\text { pipeline }\end{array}$ & P2G \\
\hline 1 & $\begin{array}{l}2-3(1), 2-6(1), 4-5(2) \\
4-8(2), 8-9(1), 7-10 \\
(3), 10-12(3), 11-12(1)\end{array}$ & $\begin{array}{l}1-5(3), 2-7(3), 4- \\
5(1), 4-8(2), 5- \\
6(1), 7-8(2), 8-9(1) \\
7-10(1)\end{array}$ & / \\
\hline 2 & $\begin{array}{l}2-6(1), 3-6(1), 4-5(2) \\
4-8(2), 8-9(1), 7-10 \\
(2), 10-12(2), 11-12(1)\end{array}$ & $\begin{array}{l}1-5(2), 2-7(2), 4- \\
5(1), 4-8(2), 5- \\
6(1), 7-8(2), 8-9(1) \\
7-10(1)\end{array}$ & $6(2)$ \\
\hline 3 & $\begin{array}{l}2-6(1), 3-6(1), 4-5(1) \\
4-8(2), 8-9(1), 7-10 \\
(1), 10-12(1), 11-12(1)\end{array}$ & $\begin{array}{l}\text { 1-5(1), 2-7(1),4- } \\
5(1), 4-8(1), 5-6(1) \\
7-8(1), 8-9(1), 7- \\
10(1)\end{array}$ & $6(3)$ \\
\hline
\end{tabular}

The results of the investment power configuration, capacity configuration, and the cost of each part of the electricity storage and gas storage system are obtained by the solution, as shown in Table 4.

Table 4. Example result

\begin{tabular}{|c|c|c|c|}
\hline Result parameters & Scene 1 & $\begin{array}{c}\text { Scene } \\
2\end{array}$ & Scene 3 \\
\hline Storage power rating / kw & 1 & I & 478.95 \\
\hline $\begin{array}{l}\text { Rated storage capacity / } \\
\qquad(\mathrm{KWH})\end{array}$ & 1 & / & 679.73 \\
\hline $\begin{array}{l}\text { Gas storage output power / } \\
\text { kw }\end{array}$ & l & l & 382.67 \\
\hline $\begin{array}{l}\text { Rated gas storage capacity / } \\
\qquad(\mathrm{KWH})\end{array}$ & I & I & 469.92 \\
\hline $\begin{array}{l}\text { Annual investment cost / } \\
10000 \text { yuan }\end{array}$ & 90.35 & 87.66 & 91.67 \\
\hline $\begin{array}{c}\text { Maintenance cost / } 10000 \\
\text { yuan }\end{array}$ & 14.69 & 13.78 & 12.63 \\
\hline $\begin{array}{l}\text { Cost of purchasing electricity } \\
\text { and gas / } 10000 \text { yuan }\end{array}$ & 37.43 & 31.79 & 21.57 \\
\hline Total cost / 10000 yuan & 142.47 & 133.23 & 125.87 \\
\hline
\end{tabular}

Compared with scenario 1 , scenario 2 verifies that the power gas collaborative planning through P2G interconnection has economic advantages. Compared with scenario 1 , the power supply of electric network node 3 is changed from line 2-3 to node 6 with P2G; in terms of equipment capacity, the connection of $\mathrm{P} 2 \mathrm{G}$ makes it more 
flexible to convert electricity into gas, which can convert the power in low price period into natural gas, so that the line capacity of power lines 7-10 and 10-12 is reduced, and the capacity of pipeline 1-5,2-7 in natural gas network is reduced; in terms of cost, $\mathrm{P} 2 \mathrm{G}$ is more flexible The introduction of equipment will add the investment cost of P2G, but the capacity of lines and pipelines will be reduced. The overall performance is to reduce the annual investment cost of 26900 yuan and the maintenance cost of 9100 yuan. The purchase cost from the superior power grid and gas network will be reduced by 56400 yuan, and the total cost will be reduced by 92400 yuan.

Compared with scenario 2, scenario 3 verifies that the coordinated planning of electricity, gas and storage can further realize economic advantages and make the system more flexible. Compared with scenario 2, the line connection between electric network node and natural gas network node remains unchanged; in terms of equipment capacity, due to the access of power storage and gas storage equipment, the time of energy supply can be changed, and the electric energy in the low price period can be stored and the load can be supplied in the peak price period, so that the 4-5 line capacity of power line connecting $\mathrm{P} 2 \mathrm{G}$ equipment in the calculation results is reduced The capacity of 1-5, 2-7 and 7-8 pipelines in the natural gas network is also reduced; in terms of cost, the introduction of power storage and gas storage equipment will add energy storage investment cost, which will increase the total investment cost by 40100 yuan. However, due to the access of energy storage, the time of energy supply can be changed, low-cost electricity can be used more flexibly, and the purchase cost from the superior power grid and gas network will be reduced by 102500 yuan, The total cost decreased by 58900 yuan.

\section{Conclusion}

Based on the analysis of the characteristics and topological structure of the components in the regional integrated energy system, a planning method for the power gas storage regional integrated energy system is proposed. The main research results are as follows

1) The influence of energy storage on the electric gas coupling system is analyzed, and the electric gas energy storage hub structure is established.

2) The constraints of each component in the regional integrated energy system are established, and the coordinated planning model of electricity, gas, and storage is proposed with the lowest cost as the goal.

3) It is verified that the collaborative planning of electricity gas network coupling has more economic advantages than the single planning and that the access of energy storage can further reduce the cost and improve the flexibility of the system, which realizes the collaborative planning of electricity gas storage system.

The power gas storage planning method proposed in this paper lays a foundation for the follow-up research, and more complex network topology and energy forms will be considered in future research.

\section{Acknowledgments}

This work is supported by Technology Project "Research on key technologies of grid side distributed energy storage optimization in different application scenarios" of State Grid Zhejiang Jiashan Country Power CO.LTD. (2020KJLH-JS-001)

\section{References}

1. Han Luying, Xu Yong., 2019 (6), Short term load forecasting based on support vector machine [J]. Computer programming skills and maintenance, 1618.

2. Zhuo ZeYing, Cao Qian, Li Qing. 2019, Wind power short-term forecasting based on empirical wavelet transform and extreme learning machine with kernels $\operatorname{method}[\mathrm{J}]$. Electrical Measurement \& Ins trumentation, 56(2): 83-89.

3. Wang Mingming, Zhao Lei, Lu fumu, et al. 2018, Millisecond precision loa d control system based on stability control technology [J]. Power supply, 35(6): 53-59.

4. KOO B G, KIM M S, KIM K H, et al. 2013, Shortterm electric load forcasting using data mining technique[C]//2013 7th International Conference on Intelligent Systems and Control (ISCO), Jan 4-5, Coimbatore, India: 153-157

5. Tan Fenglei, Chen Mengtao, Wang longlong. 2018, Short term power load forecasting based on accumulated temperature effect and optimized support vector machine [J]. DSM, 20 (5): 33-36.

6. Yu Xiaodan, Xu Xiandong, Chen Shuoyi, et al. 2016, A brief review of integrated energy system and energy Internet[J]. Transactions of China Electro Technical Society, 31(01):1-13.

7. BIE Zhaohong, WANG Xu, HU Yuan. 2017, Review and prospect of planning of energy internet[J]. Proceedings of the CSEE, 37(22):6445-6462.

8. Jia Hongjie, WANG Dan, Xu Xiandong, et al. 2015, Research on some key problems related to integrated energy system $[\mathrm{J}]$. Automation of Electric Power Systems, 39(07):198-207.

9. Zeng Ming, Liu Yingxin, Zhou Pengcheng, et al. 2018, Review and prospect of integrated energy system modeling and benefit evaluation[J]. Power System Technology, 42(06):1697-1708.

10. Huang Guori, Liu Jiawei, Wen Fushuan, et al. 2016, Collaborative planning of integrated electricity and natural gas energy systems with power-to-gas stations[J]. Electric Power Construction, 37(09):1-13.

11. Liu Tianqi, Zeng Hong, HU Chuan, et al. 2019, Planning of integrated gas and electricity system considering coordinated expansion of power-to-gas facilities and wind farms[J]. Electric Power Automation Equipment, 39(08):144-151. 